\title{
ASTROD, ASTROD I and their gravitational-wave sensitivities
}

\author{
Wei-Tou Ni ${ }^{1,2,3}$, Sachie Shiomi ${ }^{3}$ and An-Chi Liao ${ }^{3}$ \\ ${ }^{1}$ Purple Mountain Observatory, Chinese Academy of Sciences, Nanjing \\ ${ }^{2}$ National Astronomical Observatories, Chinese Academy of Sciences, Beijing \\ ${ }^{3}$ Department of Physics, National Tsing Hua University, Hsinchu
}

\begin{abstract}
ASTROD (Astrodynamical Space Test of Relativity using Optical Devices) is a mission concept with three spacecraft - one near L1/L2 point, one with an inner solar orbit and one with an outer solar orbit, ranging coherently with one another using lasers to test relativistic gravity, to measure the solar system and to detect gravitational waves. ASTROD I with one spacecraft ranging optically with ground stations is the first step toward the ASTROD mission. In this paper, we present the ASTROD I payload and accelerometer requirements, discuss the gravitational-wave sensitivities for ASTROD and ASTROD I, and compare them with LISA and radio-wave Doppler-tracking of spacecraft.

E-mail: wtni@pmo.ac.cn

PACS numbers: 04.80.Nn, 04.80.-y, 95.55.Ym,
\end{abstract}

\section{Introduction}

A standard implementation of ASTROD is to have two spacecraft in separate solar orbit carrying a payload of a proof mass, two telescopes, two 1-2 W lasers, a clock and a drag-free system, together with a similar L1/L2 spacecraft (Fig. 1)[1, 2]. The three spacecraft range coherently with one another using lasers. Simulation with timing error $50 \mathrm{ps}$ and accelerometer error $10^{-13} \mathrm{~m} / \mathrm{s}^{2}(\mathrm{~Hz})^{1 / 2}$ at frequency $f \sim 100 \mu \mathrm{Hz}$ for 1050 days gives the uncertainties of determining relativistic parameters $\gamma$ and $\beta$ to be $4.6 \times 10^{-7}$ and $4.0 \times 10^{-7}$, respectively, and that for solar quadrupole parameter $J_{2}$ to be $1.2 \times 10^{-8}[3]$. At present, satellite laser-ranging timing error has reached $5 \mathrm{ps}$. LISA's launching goal in 2012 for accelerometer noise requires $3 \times 10^{-15} \mathrm{~m} / \mathrm{s}^{2}(\mathrm{~Hz})^{1 / 2}$ at $f \sim 100 \mu \mathrm{Hz}$ [4]. Since ASTROD will be after 2012, a factor of 3 improvement on LISA goal will make 2 orders of magnitude improvement for acceleration noise in the simulation. These will put the uncertainties of measuring $\gamma$ and $\beta$ in the $10^{-9}$ to $10^{-8}$ range, and that of $J_{2}$ in the $10^{-10}$ to $10^{-9}$ range. This demands post-post-Newtonian ephemeris framework to be established for the analysis and simulation of data. Efforts have been started in this direction by colleagues [5-7].

ASTROD I with one spacecraft interferometric ranging and pulse ranging with ground stations is the first step for a full ASTROD mission [8]. The goals are testing relativity with gamma measured to $10^{-7}$, measuring solar-system parameters more precisely and improving the sensitivity for gravitational wave detection using radio Doppler tracking. The spacecraft is to be launched to encounter Venus twice to achieve a shorter period for a sooner measurement of Shapiro time delay (Fig. 2). 


\section{ASTROD sensitivity}

The algorisms for unequal arm noise cancellation of Armstrong, Estabrook \& Tinto [9, 10], and of Dhurandhar, Nayak \& Vinet [11] can readily be applied to ASTROD. For the 3-spacecraft configuration as in LISA and as in Fig. 1, they have demonstrated that one can combine the six measured time series of Doppler shifts of the one-way laser beams between spacecraft pairs, and the six measured shifts between adjacent optical benches on each spacecraft, with suitable time delays, to cancel the laser frequency fluctuations, and opical-bench vibration noise. The achievable strain sensitivity for these combinations is set by the drag-free proof-mass accelerations inside optical benches, and by the shot noise at photodetectors. The implementation of these alogarisms for ASTROD requires the armlengths known accurately to about $100 \mathrm{~m}$ and the synchronization good to about $300 \mathrm{~ns}$. Since ASTROD is an astrometry mission, the armlengths and synchronizations will be measured and implemented to better than 10 ps accuracy. Therefore, ASTROD can easily accommodate the noise cancellation algorisms even when shot noise and acceleration noise are improved significantly.

For 1-2 W laser emitting power, the shot noise sensitivity floor in the strain is $10^{-21} /(\mathrm{Hz})^{1 / 2}$ independent of armlength. With the same power, the ASTROD sensitivity would be shifted to lower frequency by a factor up to 60 (30 in average) if other frequency-dependent requirements can be met. The antenna response will be shifted correspondingly. If the accelerometer noise response can be shifted, i.e., the low-frequency response becomes better, we may have the ASTROD (2) sensitivity curve of Fig. 1 as given in [12]. ASTROD has varying armlength and the sensitivity should be in a band and integration should be performed. If the LISA accelerometer noise goal is taken, the sensitivity at low frequency is about 30 times better than LISA as indicated in ASTROD (1) in Fig. 1, as given by Rüdiger [13]. Since ASTROD is in a time frame later than LISA, if the absolute metrological accelerometer / inertial sensor can be developed, there is a potential to reach ASTROD (3) sensitivity curve. With a better lower-frequency resolution, the confusion limit for LISA should be lower for ASTROD. The modulations of signals due to orbit motions of ASTROD spacecraft, especially the radial motions, will help to distinguish gravity-gradient signals due to solar oscillation from gravitational-wave signals from outside the solar system [2].
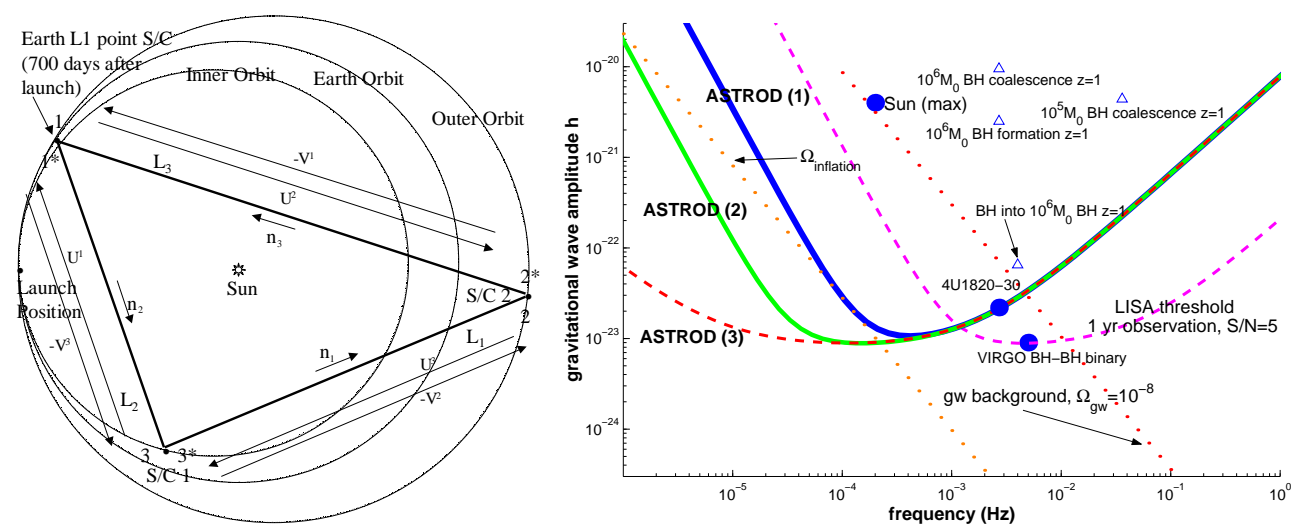

Figure 1. (left) A schematic ASTROD configuration;(right)the gravitational sensitivity curve of ASTROD and the strength of various sources. 


\section{ASTROD I payload, requirement and sensitivity}

A schematic payload configuration of ASTROD I is as in Fig. 2 [14]. The cylindrical spacecraft with diameter $2.5 \mathrm{~m}$ and height $2 \mathrm{~m}$ has its surface covered with solar panels. In orbit, the cylindrical axis is perpendicular to the orbit plane with the telescope pointing toward the ground laser station. The effective area to receive sunlight is about $5 \mathrm{~m}^{2}$ and can generate over $500 \mathrm{~W}$ of power. The total mass of spacecraft is $300-350 \mathrm{~kg}$. That of payload is $100-120 \mathrm{~kg}$ with science data rate $500 \mathrm{bps}$. The crucial technologies include (i) $100 \mathrm{fW}$ weaklight phase locking [15]; (ii) design and development of sunlight shield [8]; (iii) design and development of drag-free system. The requirements for the drag-free system are less stringent than LISA. In Table 1 we provide a summary of the parameter values that meet the accelerometer requirements for ASTROD I, in comparison with LISA. We use the notations and the LISA values of Schumaker [16]. The listed values are for the frequency at $0.1 \mathrm{mHz}$. The parameter values are largely relaxed for ASTROD I. The same values satisfy requirements for higher-frequency range, where frequency-dependent contributions to the acceleration noise become smaller.

A typical design orbit for August 4, 2010 launch is shown on the left of Fig 2: two encounters with Venus to swing the spacecraft to the other side of the Sun to conduct Shapiro time delay measurement and to measure Venus multipole moments [17]. Assuming a $10 \mathrm{ps}$ timing accuracy and $10^{-13} \mathrm{~m} / \mathrm{s}^{2}(\mathrm{~Hz})^{1 / 2}$ (at $f \sim 100 \mu \mathrm{Hz}$ ) inertial sensor/accelerometer noise, our simulation of the accuracy for determining the relativistic parameters $\gamma$ and $\beta$, and the solar quadrupole parameter $\mathrm{J}_{2}$ gives $10^{-7}, 10^{-7}$ and $10^{-8}$, respectivly, for their uncertainties [17].

Like the radio Doppler tracking of spacecraft [18], ASTROD I also has sensitivity to low-frequency gravitational-wave. Clock noise and the propagation noise are the dominant noise sources. Clock noise is the dominant instrumental noise. As clock stability improves, this noise becomes smaller. The propagation noise is due to fluctuations in the index of refraction of the troposphere, ionosphere and the interplanetary solar plasma. The fluctuations due to ionosphere and interplanetary plasma are not important for laser ranging. Tropospherical effect can be substracted
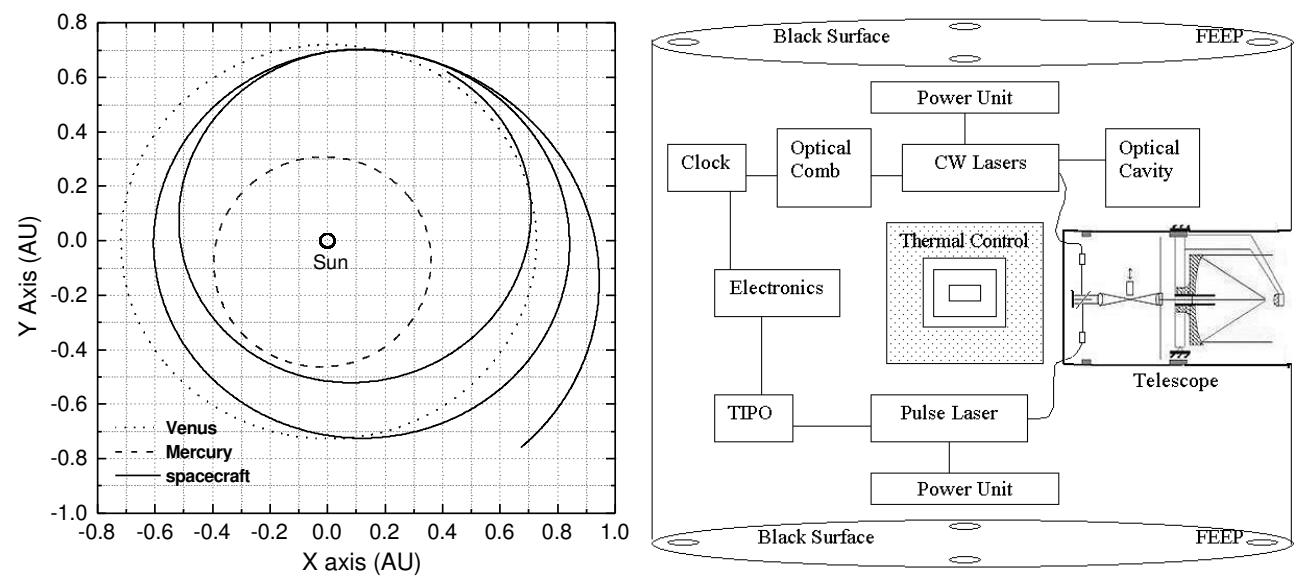

Figure 2. (left) A design orbit of ASTROD I in the heliocentric ecliptic coordinate system;(right)a schematic diagram of payload configuration of ASTROD I. 
Table 1. Parameter values that meet ASTROD I accelerometer requirements, as compared with LISA.

\begin{tabular}{|c|c|c|}
\hline$f=(2 \pi)^{-1} \omega=0.1 \mathrm{mHz}$ & ASTROD I & LISA \\
\hline Acceleration noise goal at $0.1 \mathrm{mHz}: A_{p}\left[\mathrm{~m} \mathrm{~s}^{-2} \mathrm{~Hz}^{-1 / 2}\right]$ & $10^{-13}$ & $3 \times 10^{-15}$ \\
\hline \multicolumn{3}{|l|}{ Parameter values } \\
\hline \multicolumn{3}{|l|}{ Proof Mass (PM) } \\
\hline Magnetic susceptibility: $\chi_{m}$ & $10^{-5}$ & $10^{-6}$ \\
\hline Maximum charge build-up: $q[\mathrm{C}]$ & $10^{-12}$ & $10^{-13}$ \\
\hline Residual gas pressure: $P[\mathrm{~Pa}]$ & $10^{-5}$ & $10^{-6}$ \\
\hline $\begin{array}{l}\text { Fluctuation of temperature difference across } \\
\text { PM and housing: } \delta T_{d}\left[\mathrm{~K} \mathrm{~Hz}^{-1 / 2}\right] \text { (at } 0.1 \mathrm{mHz} \text { ) }\end{array}$ & $1.4 \times 10^{-3}$ & $2.2 \times 10^{-5}$ \\
\hline \multicolumn{3}{|l|}{ Spacecraft (SC) } \\
\hline Thruster noise $\left[\mu \mathrm{N} \mathrm{Hz}^{-1 / 2}\right]($ at $0.1 \mathrm{mHz})$ & 0.5 & 0.1 \\
\hline $\begin{array}{l}\left.\text { Fluctuation of temperature in } \mathrm{SC}: \delta T_{s c}\left[\mathrm{~K} \mathrm{~Hz}^{-1 / 2}\right] \text { (at } 0.1 \mathrm{mHz}\right) \\
\text { Capacitive sensing }\end{array}$ & 0.4 & 0.004 \\
\hline $\begin{array}{l}\text { Voltage difference between average voltage across opposite } \\
\text { faces and voltage to ground: } V_{0 g}[\mathrm{~V}]\end{array}$ & 1 & 0.1 \\
\hline $\begin{array}{l}\text { Fluctuation of voltage difference across opposite faces: } \\
\delta V_{d}\left[\mathrm{~V} \mathrm{~Hz}^{-1 / 2}\right](\text { at } 0.1 \mathrm{mHz})\end{array}$ & $10^{-4}$ & $10^{-5}$ \\
\hline Asymmetry in gap across opposite sides of PM: $\Delta d[\mu \mathrm{m}]$ & 10 & 1 \\
\hline \multicolumn{3}{|l|}{ Laser power } \\
\hline Fluctuation of laser power: $\delta I\left[\mathrm{~W} \mathrm{~Hz}^{-1 / 2}\right]$ (at $0.1 \mathrm{mHz}$ ) & $2 \times 10^{-6}$ & $2 \times 10^{-8}$ \\
\hline
\end{tabular}

to a large extent by 2-color (2-wavelength) laser ranging or by using artificial stars. The ASTROD I spacecraft noise is very small and negligible. With these improvements, ASTROD I will have a couple of times to several times better sensitivity to gravitational-wave for the same spacecraft-Earth configuration. Detailed analysis will be presented in a forthcoming publication.

We thank Foundation of Minor Planets of Purple Mountain Observatory and National Science Council [Grant no. NSC 92-2112-M-007-042] for financial supports.

\section{References}

[1]Bec-Borsenberger A et al 2000 ASTROD ESA F2/F3 Mission Proposal; and references therein [2]Ni W-T 2002 Int. J. Mod. Phys. D11 947; and references therein

[3]Chiou D-W, and Ni W-T 2000 Orbit Simulation for the Determination of Relativistic and SolarSystem Parameters for the ASTROD Space Mission, 33rd COSPAR Scientific Assembly, Warsaw, 16-23 July, 2000; Chiou D-W, and Ni W-T 2000 Advances in Space Research 251259

[4]LISA: System and Technology Study Report 2000 ESA document ESA-SCI (2000) 11, July 2000, revised as ftp://ftp.rzg.mpg.de/pub/grav/lisa/sts/sts_1.05.pdf and references therein

[5]Huang T, and Tao J 2002 Int. J. Mod. Phys. D11 1011

[6]Li G, and Zhao H 2002 Int. J. Mod. Phys. D11 1021

[7]Xu C-M, and Wu X-J 2003 Chin. Phys. Lett. 20195

[8]Ni W-T et al 2002 Int. J. Mod. Phys. D11 1035; and references therein

[9]Tinto M, Estabrook F B and Armstrong J W 2002 Phys. Rev. D65 082003; and references therein

[10]Prince T A, Tinto M, Larson S L and Armstrong J W 2002 Phys. Rev. D66 122002

[11]Dhurandhar S V, Nayak K R,Vinet J-Y 2002 Phys. Rev. D65 102002

[12]Ni W-T 1997 Gravitational Wave Detection 117 eds. Tsubono K et al (Tokyo: UAP)

[13]Rüdiger A 2002 Int. J. Mod. Phys. D11 963

[14] ASTROD I Pre-Phase A Report 2003 in preparation

[15]Liao A-C, Ni W-T, and Shy J-T 2002 Int. J. Mod. Phys. D11 1075

[16]Schumaker B L 2003 Class. Quantum Grav. 20 S239; and references therein

[17]Tang C J et al 2003 Orbit Design for the ASTROD I paper in preparation

[18]Tinto M 2002 Class. Quantum Grav. 19 1767; and references therein 